\title{
The cardiac electrical status of a patient with mithocondrially inherited disease - case report
}

\author{
Delia Nebunu ${ }^{*, 1}$, Juan Antonio Sieira Rodríguez-Moret², Rubén Casado-Arroyo² \\ 1 "Grigore T. Popa" University of Medicine and Pharmacy, lasi, Romania, ${ }^{2}$ Université Libre de Bruxelles, \\ Department of Cardiology, Erasme Hospital, Brussels, Belgium
}

\begin{abstract}
Cardiac complications, such as myocardial disease and arrhythmias, are frequent and may be severe in patients with mitochondrial disease. Mitochondrial diseases are a heterogeneous group of disorders that can affect multiple organs with varying severity. We hereby present the case of a 58year-old man admitted in the Cardiology Department for an Electrophysiological study asked by his General practitioner in the context of a past medical history evident for a mitochondrial cytopathy and subsequent to arrhythmic symptomatology revealed as uncomfortable palpitations and to disclosure of Holter bursts of supraventricular tachycardia.
\end{abstract}

Keywords: supraventricular tachycardia, electrophysiological study, ablation, Leber disease

\section{Introduction}

Pre-excitation syndrome is common in families with Leber hereditary optic neuropathy (LHON) [1]. It is a primary mitochondrial DNA (mtDNA) disorder in the general population, with an estimated prevalence of 1:39.000 in the Netherlands. It is an important cause of severe, usually irreversible, visual loss among young adults with a peak age of onset in the second and third decades of life, transmitted in a nonMendelian or maternal pattern [2]. Mutations in genes that direct synthesis of ATP by oxidative phosphorylation can cause pleomorphic diseases, given that mutations are heteroplasmic and different tissues have variable energy needs [3]. Due to visual impairment and decreased hearing acuity, taking the case history proved difficult. Consequently, we were not able to trace the family history.

Received: August 2014; Accepted after review: September 2014; Published: September 2014

*Corresponding author: Delia Nebunu, "Grigore T. Popa" University of Medicine and Pharmacy, 16 Universitatii St, 700115 lasi, Romania. Email: delia neb@yahoo.co.uk
The aim of this case report is to stress that the mitochondrial disease, clinically variable, can affect the heart whose activity relies heavily on this particular organelle, hence the link between [4].

\section{Case presentation}

We present the case of a 58-year-old man admitted in the Cardiology Department of Erasme Hospital of Brussels, for an Electrophysiology study asked by his General practitioner in the context of a past medical history evident for a mitochondrial cytopathy and subsequent to arrhythmic symptomatology, revealed as unconfortable palpitations, and to disclosure of Holter bursts of supraventricular tachycardia.

The patient reported a moderate dyspnea (class III according to NYHA scale) gradually developed, and diffuse pain all over the body. Apart from the chief complain, there were no other cardiovascular matters. In examination, the patient showed global development delay, amyotrophy, and distended abdomen. Past medical history included an idiopathic 
cardiomyopathy when he was 3 month-old, one eye visual loss when he was 21 year-old, progressive visual loss to the controlateral eye, progressive hearing loss after onset of the optic atrophy, arterial hypertension, coronary artery by-pass at the age of 35 , and diabetes mellitus type II.

In regard to cardiovascular risk factors, the patient was a smoker ( 1 pack of cigarettes per day for 10 years), chronic alcoholic, diabetic, sedentary, with hypercholesterolemia and arterial hypertension. As for family history, although highly suspected in regard to a genetic disease, the patient stated that there were no particularities.

Affected individuals are often aware of other affected family members, but up to $40 \%$ have no family history [5]. It is the case a more likely difficult to trace family history, given that de novo mutation is rare in LHON [6].

\section{Results}

An electrophysiological study was performed (Figure 1). Baseline rhythm was sinus with no evident preexcitation. Ventricular pacing maneuvers demonstrated concentric and decremental conduction. Atrial pacing maneuvers were compatible with anterograde conduction over the AV node. No dual AV node physiology was demonstrated.

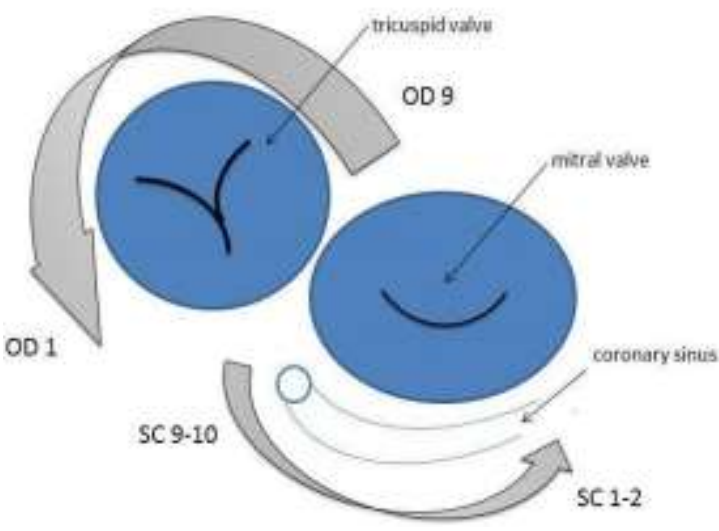

Fig. 1. Position of the electrodes: OD 1-9 around tricuspid valve (OD - oreillet droit, right atrium) and SC 1-2 $\rightarrow$ 9-10 around the coronary sinus (SC sinus coronaire, coronary sinus)

Under isoproterenol infusion and atrial burst, a narrow QRS tachycardia was induced, with cycle length of $360 \mathrm{~ms}$. Pacing maneuvers were diagnostic of atrial tachycardia (VAAV sequence after ventricular pacing). Earliest activation was found at the coronary sinus ostium (Figure 2 and Figure 3 ).

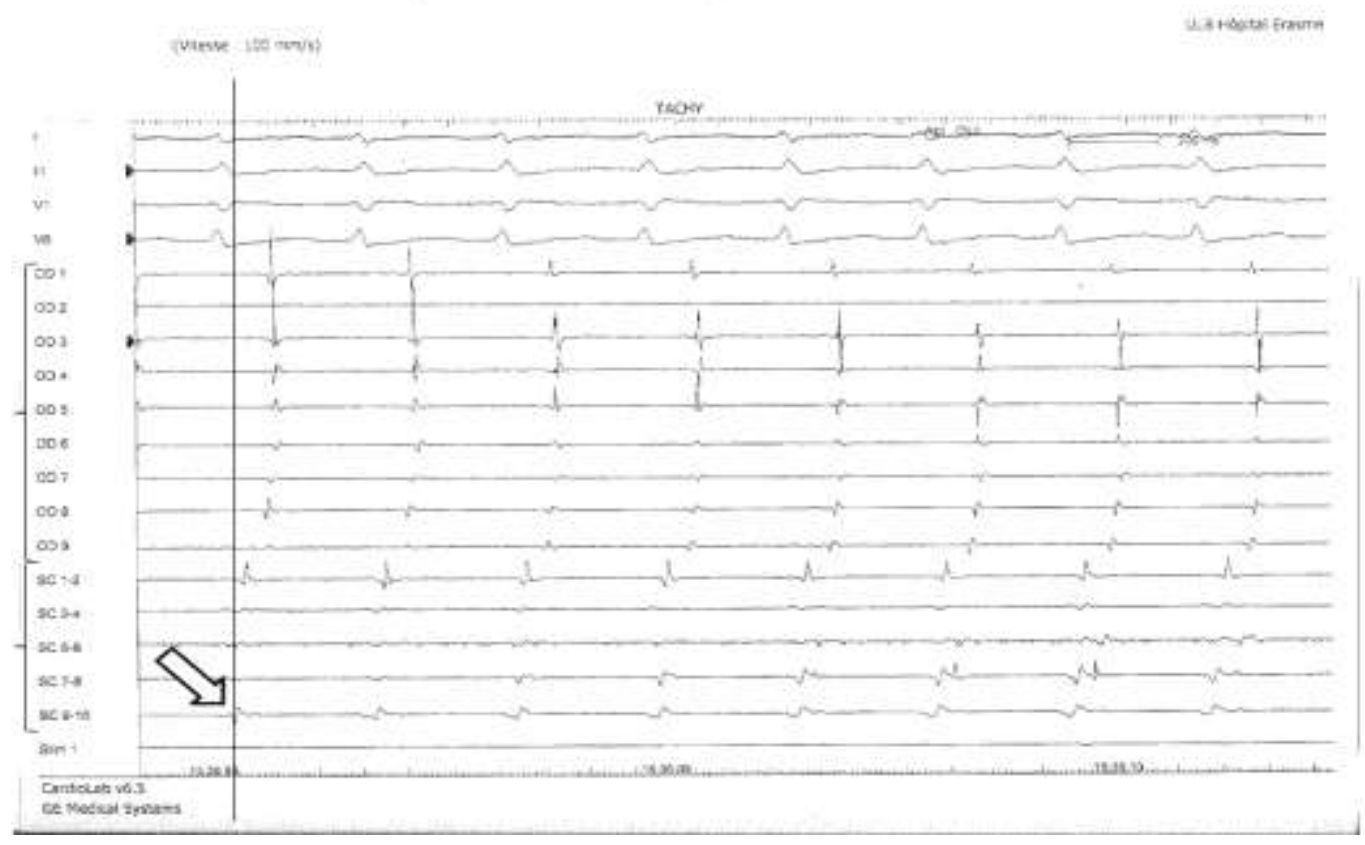

Fig. 2. Atrial tachycardia with cycle length of 320 milliseconds. Earliest conduction observed in SC 9-10 corresponding with the ostium of the coronary sinus 


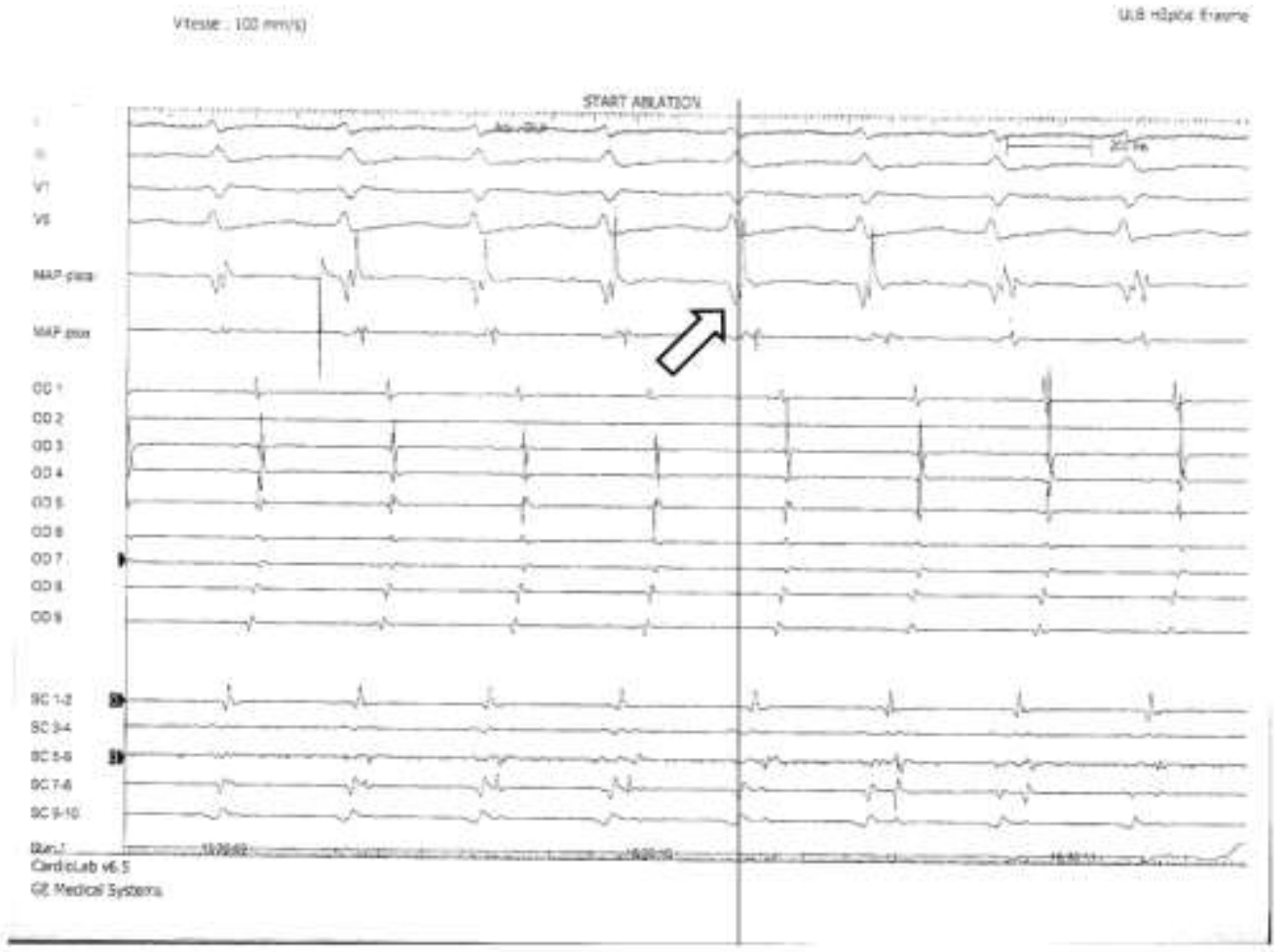

Fig. 3. Atrial tachycardia with cycle length of 320 miliseconds. Mapping catheter at the ostium of the coronary sinus showing the earliest activation at this point.

Ablation of the region with radiofrequency energy (power $50 \mathrm{~W}$ and temperature $55^{\circ} \mathrm{C}$ ) was followed by interruption of the arrhythmia during the first seconds of application. After it induction of the tachycardia was not possible (Figure 4).

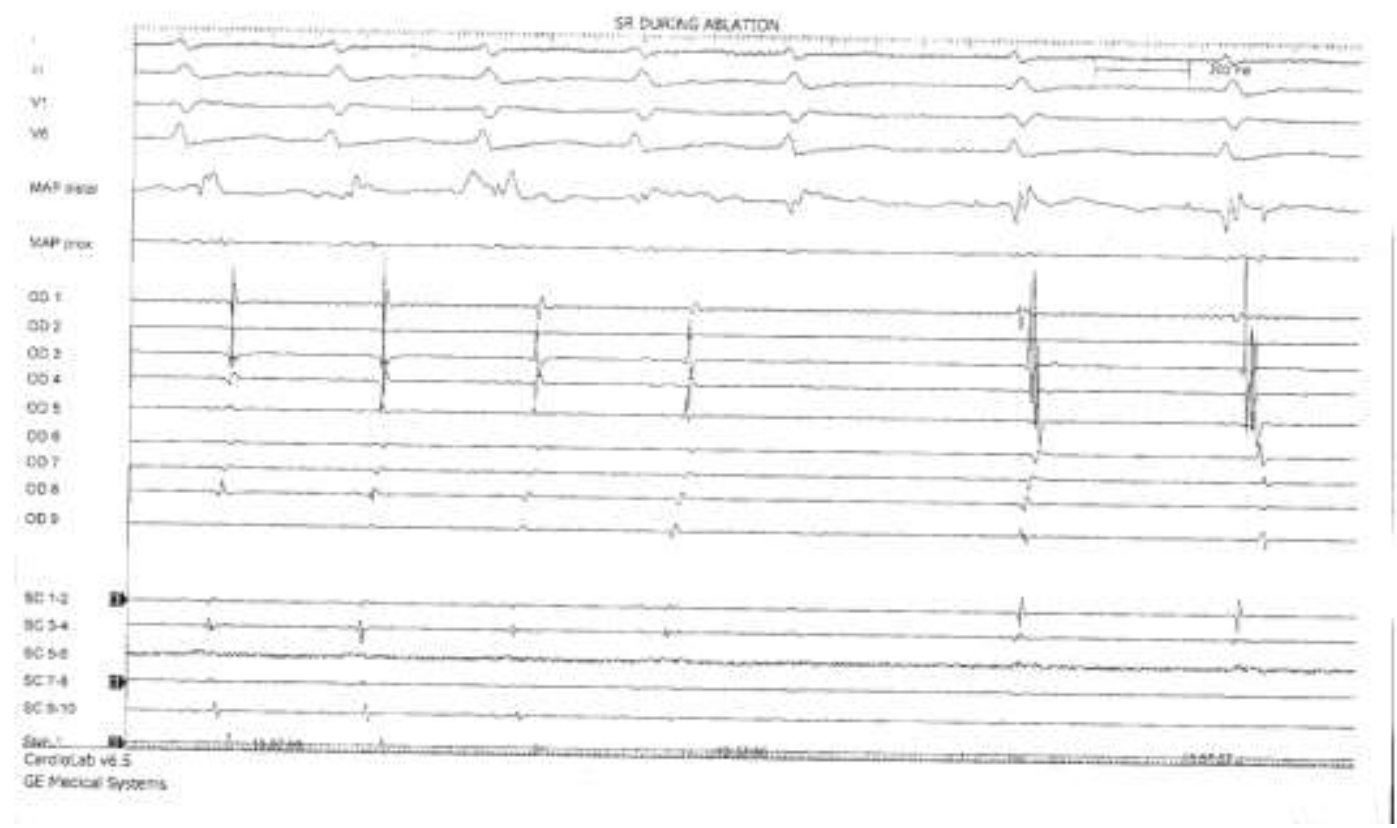

Fig. 4. Reversion to sinus rhythm during ablation at the selected point 


\section{Discussions}

Management of individuals affected by Leber's disease implies multidisciplinary approach.

The treatment of manifestations is largely supportive, considered to minimize the functional consequences of the mitochondrial cytopathy, as investigations into potential therapies are still in their nascence (antiapoptotic agents and a variety of gene therapies).

Although visual failure is the defining clinical feature in this mitochondrial genetic disorder, cardiac arrhythmias and neurologic abnormalities have been reported to be more common in individuals with LHON than in controls [7]. Moreover, treatment of cardiac arrhythmias is encouraged, especially in

\section{References}

1. Nikoskelainen E, Wanne O, Dahl M. Preexcitation syndrome and Leber's hereditary optic neuroretinopathy. Lancet 1985; 1(8430):696.

2. Man PY, Turnbull DM, Chinnery PF. Leber hereditary optic neuropathy. J Med Genet 2002; 39:162-9.

3. Bernier FP, Boneh A, Dennett X. Diagnostic criteria for respiratory chain disorders in adults and children. Neurology 2002; 59:1406-1411.

4. Wahbi K, Larue S, Jardel C, et al. Cardiac involvement is frequent in patients with the m.8344A $>$ G mutation of mitochondrial DNA. Neurology 2010; 74:674-677.

5. Harding AE, Sweeney MG, Govan GG, RiordanEva P. Pedigree analysis in Leber hereditary symptomatic patients, as it improves the quality of life.

\section{Conclusion}

Following a disfunction in the mitochondrial respiratory chain, electrical conduction system can be disturbed, leading to variable arrhythmias [7].

In the context of Leber's disease, the patient had a supraventricular tachycardia, clinically symptomatic, diagnosed on the basis of a Holter-monitoring.

During the burst pacing in the electrophysiological study, an atrial focal tachycardia was induced. It was mapped in the right atrium, near the ostium of the coronary sinus and it was ablated by radiofrequency.

optic neuropathy families with a pathogenic mtDNA mutation. Am J Hum Genet 1995; 57:7786.

6. Biousse V, Brown MD, Newman NJ, et al. De novo 14484 mitochondrial DNA mutation in monozygotic twins discordant for Leber's hereditary optic neuropathy. Neurology 1997; 49:1136-8.

7. Robert M, Zipes D. Mechanisms of arrhythmogenesis. In Bonow RO, Mann DL, Zipes D, Braunwald's Heart Disease: A Textbook of Cardiovascular Medicine, $9^{\text {th }}$ Edition, Philadelphia: Elsevier Saunders 2012; 672-678. 